EPJ Web of Conferences 81, 05011 (2014)

DOI: $10.1051 /$ epjconf/20148105011

(C) Owned by the authors, published by EDP Sciences, 2014

\title{
The Meson Spectroscopy Program Using the Forward Tagger with CLAS12 at Jefferson Lab
}

\author{
Stuart Fegan ${ }^{1, a}$ \\ for the CLAS Collaboration \\ ${ }^{1}$ Istituto Nazionale di Fisica Nucleare (INFN), Sezione di Genova, Via Dodecaneso 33, Genova, 16146, Italy
}

\begin{abstract}
The $12 \mathrm{GeV}$ upgrade to the Continuous Electron Beam Accelerator Facility (CEBAF) will enable a new generation of experiments in hadronic nuclear physics at Jefferson Lab, seeking to address fundamental questions in QCD. For example, confirming the existence of exotic states, suggested by both quark models and lattice calculations, would allow gluonic degrees of freedom to be explored, and may help explain the role played by gluons in the QCD interaction.

In Experimental Hall B, meson spectroscopy (the MesonEx experiment) will be performed using low $\mathrm{Q}^{2}$ electron scattering to produce quasi-real photons. The scattered electron is detected at small angles by the Forward Tagger device, determining the properties of the photon on an event-by-event basis. This technique has notable advantages over real photon beams, and over hadronic beam experiments, where most experimental data exists.

This article will focus on the development of the Forward Tagger by the INFN Genova group, and the proposed MesonEx experiment.
\end{abstract}

\section{Introduction}

From its roots in the experimental particle physics of the 1950s and 60s, the development around 40 years ago of the theory of Quantum Chromodynamics (QCD) has served as a powerful tool in our understanding of strong force interactions, confinement, and the structure of hadrons. At sufficiently high energies, perturbation theory is able to describe the sub-nucleon interactions governed by QCD in the same manner in which the photon and electron interactions are described in Quantum Electrodynamics (QED). However, at the lower energies of the quark-hadron interface, the running coupling constant of QCD approaches unity, and perturbative approaches can no longer accurately describe the underlying processes. QCD then enters the non-perturbative regime, where rigorous solutions are not yet available.

Quark models have played a vital role in the non-perturbative energy regime, predicting numerous hadronic states from the degrees of freedom associated with the coloured quarks of QCD. Many of these states have been observed and studied experimentally, with extensive details of their properties well-known [1]. However, the quark model only tells part of the story and further studies, encompassing theory, phenomenology, and experimental observations using a new generation of facilities, will be necessary to form a more complete picture.

\footnotetext{
a e-mail: fegan@ge.infn.it
} 


\section{Hadron Spectroscopy}

In spite of the successes of QCD, there are still gaps in our understanding of the dynamics of the QCD interaction, and in how quarks and gluons are confined in hadronic states. For example, the total mass of the valence quarks in a nucleon only accounts for around $1 \%$ of the observed nucleon mass; the rest is generated dynamically by strong interactions in the nucleon, which are governed by QCD.

Understanding the known issues in QCD requires detailed study of the properties of hadrons and the rules of QCD, and hadron spectroscopy is one such tool that seeks to achieve this. By studying the hadron spectrum, it is hoped that some of the fundamental questions in this field can be answered, including learning about the internal structure and internal degrees of freedom of the hadrons, understanding the role of gluons, exploring the origin of quark confinement, and ascertaining whether three quark and quark-antiquark are the only possible configurations.

\subsection{Meson spectroscopy}

Mesons, being composed of a quark and antiquark, are the simplest bound quark system, making them an obvious choice for studies of how quarks combine to form hadrons. At low mass, constituent quark models have had some success at describing the meson spectrum. By considering mesons as quark-antiquark pairs, the combinations of spin, S, and orbital angular momentum, L, can describe sets of states with the same quantum numbers $J^{P C}$. As a consequence of SU(3) flavour symmetry, a nonet of states with the same $J^{P C}$ quantum numbers is implied for each value of $\mathrm{L}$ and $\mathrm{S}$.

However, despite the success of quark model approaches at predicting low mass states, many of the model predictions for higher mass mesons have yet to be observed. Even among the observed states, there remains uncertainty as to precisely where they fit into the quark model. The $f_{0}(980)$, for example, does not have a clear assignment yet, and lying close to the $\mathrm{K} \overline{\mathrm{K}}$ threshold complicates its experimental determination. This leaves its QCD nature somewhat ambiguous [2].

These ambiguities in resolving observed states to the quark model may be due to limitations of experimental techniques, problems with the models, or the existance of states not considered by the quark model, including hybrids and glueballs.

\subsubsection{Unconventional states}

One of the fundamental principles of QCD is that bound states should be color neutral, however this does not mean that the well-known configurations of two or three quarks are the only states that can

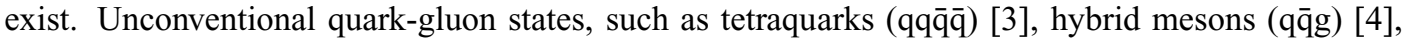
and glueballs [5] are perfectly allowable within QCD, and spectroscopy of these states would enable exploration of the gluonic degrees of freedom in a way that has not yet been possible.

Although the existence of these states has yet to be confirmed unambiguously, they are predicted by phenomenological models, which are also able to suggest possible masses and decay modes.

\subsection{Lattice QCD}

Until recently, the quark model has been the primary tool for predicting the hadronic spectrum and making estimates of their properites. However, with recent developments in the field of Lattice QCD, this is no longer the case, and lattice simulations are now making an important contribution.

Although lattice predictions are constrained by the use of unphysical quark masses, results of such simulations can be interpreted in quark model terms as predicting a number of unobserved states, including unconventional states $[6,7]$. Even at these unphysical masses, the results show qualitatively similar spectra across a range of quark masses, suggesting that at the known quark masses of the real world, analogous states may exist. 
MESON 2014-13 ${ }^{\text {th }}$ International Workshop on Production, Properties and Interaction of Mesons

\subsection{Hybrids and exotics}

Theory and phenomenology now point towards the existence of a rich spectrum of unconventional states, and experimental searches for such states are now being considered. It is thought that hybrids with exotic quantum numbers, i.e. quantum numbers forbidden in simple quark models, may be more effectively produced by photon beams than with a pion beam [8], and it is in this area that the Jefferson Lab meson spectroscopy program will contribute.

\section{Jefferson Lab, CEBAF, and the $12 \mathrm{GeV}$ Upgrade}

Jefferson Lab, a US Department of Energy national facility, located in Newport News, Virginia, is host to the Continuous Electron Beam Accelerator Facility (CEBAF), a Superconducting Radio-Frequency (SRF) accelerator in a two-linac, multi-pass, racetrack configuration. An extensive upgrade to the accelerator, involving a near-doubling of the beam energy to $12 \mathrm{GeV}$ and the construction of a fourth experimental hall, is nearing completion. Commissioning of the beam, as well as that of the new and upgraded detector systems in the four experimental halls, is currently underway, with a staggered program that should be completed in 2016. By this time, upgraded beam will be simultaneously available to multiple halls, at varying energy and current, providing the same level of versatility in operation that hallmarked the pre-upgrade era of CEBAF.

\subsection{CLAS12 and the Forward Tagger}

In Hall B, the previously used CLAS detector [9] is being extensively rebuilt to become CLAS12 [10]. CLAS12 is a large acceptance spectrometer, combining a forward detector based upon a toroidal magnetic field with a central detector based around a solenoid. CLAS12 will provide a combination of good particle identification, particularly for charged particles, and good energy resolution, with large acceptance for the kinematics of the upgraded beam, and operating at ten times the luminosity of CLAS.

CLAS12 has been designed for a new generation of experiments in Deeply Virtual Compton Scattering (DVCS) and Deep Inelastic Scattering (DIS), but its capabilities are also useful for hadron spectroscopy, with an experiment in meson spectroscopy approved for the CLAS12 program (MesonEx) [11].

MesonEx will combine CLAS12 with the Forward Tagger device [12], to study low $Q^{2}$ electron scattering, which has been identified as an attractive technique for meson spectroscopy. These electrons are scattered at low angles, where the exchanged virtual photon is considered to be quasi-real, and detected by the Forward Tagger, which extends CLAS12 acceptance to include polar angles of $2.5^{\circ}$ to around $4.5^{\circ}$. The photons, and their inherent linear polarization, can then be reconstructed on an event-by-event basis.

\section{Partial Wave Analysis}

Simple bump-hunting is not suitable for resolving the plethora of states from each other, and from non-resonant contributions. Therefore, partial wave analysis (PWA) will be performed on this new generation of data in order to extract signals of new states.

Promising work has already been undertaken on this using CLAS data from the $6 \mathrm{GeV}$ era, with PWA performed on the $\gamma p \rightarrow p \pi^{+} \pi^{-}$reaction [13]. This analysis has seen the first evidence in photoproduction for the $f_{0}(980)$ state, with other analyses on existing CLAS data now in progress to 
enable the development of tools and techniques for the coming data at $12 \mathrm{GeV}$, where detailed study of higher mass states will be performed.

Data from the $12 \mathrm{GeV}$ program at JLab will provide high-quality, high statistics data, requiring a robust analysis framework to process and interpret. The HASPECT (Hadron SPEctroscopy CenTer) group is a collaboration of several of the groups interested in the Jefferson Lab meson spectroscopy program, from both theoretical and experimental backgrounds, who have embarked on a series of pathfinder analyses [14] on data from CLAS at $6 \mathrm{GeV}$, with the aim of developing the required tools and procedures for robust and effective analysis of data from CLAS12.

\section{Conclusions}

Meson spectroscopy poses a tantalising opportunity to address findamental questions in our understanding of QCD. The existence of exotic and unconventional states, suggested by both quark models and Lattice QCD calculations, would enable exploration of the role of gluons in the QCD interaction at the energy scale of hadrons. The planned MesonEx experimental program in meson spectroscopy with the CLAS12 detector at Jefferson Lab, aims to build on the lab's historical strengths in hadron spectroscopy, and continue this legacy into higher energies. Preparations for this program are well underway, with construction of the required detector systems at an advanced stage and development of the analysis tools and procedures seeking to outline a roadmap for effective analysis not just at Jefferson Lab, but across the spectroscopy community.

\section{References}

[1] J. Beringer et al. (Particle Data Group), Phys. Rev. D 86, 010001 (2012).

[2] D. V. Bugg, Phys. Rept. 397, 257 (2004).

[3] R. J. Jaffe, Phys. Rev. D 15, 267 (1977).

[4] C. Amsler and N. A. Törnqvist, Phys. Rept. 389, 61 (2004).

[5] W. Ochs, J. Phys. G: Nucl. Part. Phys. 40, 043001 (2013).

[6] J. J. Dudek et al., Phys. Rev. D 83, 111502 (2011).

[7] P. Guo et al., Phys. Rev. D 78, 056003 (2008).

[8] A. P. Szczepaniak and M. Swat, Phys. Lett. B 516, 72 (2001).

[9] B. A. Mecking et al., Nucl. Inst. and Meth., A 503, 513 (2003).

[10] CLAS12 Technical Design Report, http://www.jlab.org/Hall-B/clas12 tdr.pdf(2008).

[11] CLAS collaboration. Meson Spectrsocopy with low $Q^{2}$ electron scattering in CLAS12. Proposal to Jefferson Laboratory PAC37 (2011).

[12] Forward Tagger Technical Design Report, http://www.ge.infn.it/ batta/jlab/ft-tdr.2.0.pdf(2012).

[13] M. Battaglieri et al. (CLAS Collaboration), Phys. Rev. D 80, 072005 (2009).

[14] A. Celentano. The Forward Tagger detector for CLAS12 at Jefferson Laboratory and the MesonEx experiment. $\mathrm{PhD}$ thesis (2014). 